RAPID COMMUNICATION

\title{
The effect of uniaxial stress component on the lattice strains measured by a diffraction method using opposed anvil device: trigonal system
}

\author{
A K SINGH and C BALASINGH \\ Materials Science Division, National Aerospace Laboratories, Bangalore 560017, India \\ MS received 11 April 1996
}

\begin{abstract}
The equations have been derived for the trigonal system to calculate the lattice strains produced by the non-hydrostatic pressure condition which arises when the sample is compressed between the anvils without any pressure transmitting medium.
\end{abstract}

Keywords. Uniaxial stress component; lattice strains; opposed anvil device; trigonal system.

\section{Introduction}

The lattice strains as a function of pressure can be measured by recording X-ray diffraction patterns from the specimen compressed in an opposed anvil device such as a diamond anvil cell. When the specimen is compressed between the anvils without any pressure transmitting medium, the stress state at the centre of the specimen, $\sigma_{i j}$, is non-hydrostatic. In recent articles generalized theories using anisotropic elasticity theory were developed for cubic (Singh 1993) and hexagonal (Singh and Balasingh 1994) systems to calculate the lattice strains which correspond to the strains measured under nonhydrostatic pressure condition. Recently, Mao et al (1995) developed a new technique to measure the $d$-spacings as a function of $\psi$ ( $\psi$ being the angle between the diffracting plane normal and the direction of the applied load) and collected the data for the cubic and trigonal phases of Wustite. The interpretation of such data will require an expression for trigonal system to calculate the lattice strains under non-hydrostatic condition. In this article, we derive the relevant equations for the trigonal system in a form suitable for the analysis of the experimental data. The method followed in the derivation of the equations is given in detail in the earlier papers (Singh 1993; Singh and Balasingh 1994).

\section{Stress state}

The stress state, $\sigma_{i j}$, at the centre of the specimen compressed in an opposed anvil device is defined by the radial component (in the plane of the anvil face) $\sigma_{1}$ and the axial component (along the direction of the applied load) $\sigma_{3}$. The difference $\left(\sigma_{3}-\sigma_{1}\right)$ denoted by $t$, has been termed as uniaxial stress component. The stress state at the centre of the specimen is completely described by

$$
\begin{aligned}
\sigma_{i j} & =\left[\begin{array}{lll}
\sigma_{1} & 0 & 0 \\
0 & \sigma_{1} & 0 \\
0 & 0 & \sigma_{3}
\end{array}\right] \\
& =\left[\begin{array}{lll}
\sigma_{\mathrm{p}} & 0 & 0 \\
0 & \sigma_{\mathrm{p}} & 0 \\
0 & 0 & \sigma_{\mathrm{p}}
\end{array}\right]+\left[\begin{array}{rrc}
-\frac{t}{3} & 0 & 0 \\
0 & -\frac{t}{3} & 0 \\
0 & 0 & \frac{2 t}{3}
\end{array}\right]
\end{aligned}
$$




$$
=\sigma_{\mathrm{p}}+d_{i j}
$$

where $\sigma_{\mathrm{p}}=\left[2 \sigma_{1}+\sigma_{3}\right] / 3=\left(\sigma_{1}+t / 3\right)$ is the equivalent hydrostatic pressure and the $d_{i j}$ is the deviatoric stress component. As the load on the opposed anvil set-up is increased, $\sigma_{\mathrm{p}}$ increases and $t$ settles down at a value which equals the yield stress of the specimen material at a pressure $\sigma_{\mathrm{p}}$. With further increase in the applied load, $\sigma_{\mathrm{p}}$ increases rapidly; the increase in $t$ is comparatively small and corresponds to the increase of the yield stress of the specimen material with pressure. The strains produced by $\sigma_{p}$ can be very large and are better analyzed using a standard equation of state valid for large strains. The stress $d_{i j}$, being very small (as compared with $\sigma_{\mathrm{p}}$ ), produces strain $\varepsilon_{d}(h k l)$ which can be calculated using linear elasticity theory. In terms of the measured $d$-spacing, $d_{p+d}^{\text {obs }}$ under non-hydrostatic conditions can be written as

$$
\varepsilon_{d}(h k l)=\left(d_{p+d}^{\mathrm{obs}}-d_{p}\right) / d_{p} .
$$

Following the approach given in the earlier papers (Singh 1993; Singh and Balasingh 1994) it can be easily shown that,

where

$$
\varepsilon_{d}(h k l)=\left(1-3 \cos ^{2} \psi\right) F
$$

$$
F=-\left(\frac{t}{3}\right)\left[\alpha\left(2 G_{\mathrm{R}}^{X}\right)^{-1}+(1-\alpha)\left(2 G_{\mathrm{V}}\right)^{-1}\right]
$$

$G_{\mathrm{R}}^{X}$ and $G_{\mathrm{V}}$ denote the shear modulus under Reuss condition relevant for X-ray diffraction and Voigt condition respectively. It may be noted that $G_{\mathrm{R}}^{X}$ differs from the shear modulus under Reuss condition. $\alpha$ is a fraction between 0 and 1.

From (2) and (3) we get the following useful relation,

$$
d_{p+d}^{\mathrm{obs}}=d_{p}\left[1+F\left(1-3 \cos ^{2} \psi\right)\right] .
$$

\subsection{Derivation of $G_{R}^{X}$}

To calculate the lattice strain under the action of a stress field, three orthogonal coordinate systems are used. The relative orientations between these axes are shown in figure 1 . The diffracting plane $(\mathrm{ABC})$ is referred to the $x_{i}^{\prime \prime}$ axes with $x_{1}^{\prime \prime}$ and $x_{3}^{\prime \prime}$ coinciding with the crystallographic axes $a$ and $c$ and the $x_{2}^{\prime \prime}$ axis is chosen perpendicular to both $x_{1}^{\prime \prime}$ and $x_{3}^{\prime \prime}$ to form a right handed system of coordinates. The $x_{i}^{\prime}$ are the diffraction plane coordinate system with $x_{1}^{\prime}$ along $\mathrm{O}^{\prime} \mathrm{A}$ and $x_{3}^{\prime}$ along $\mathrm{OO}^{\prime}$ normal to the plane ABC. The relative orientations between the coordinate system $x_{i}$ and diffraction plane coordinate system $x_{i}^{\prime}$ can be specified by the angles $\varphi$ and $\psi$ as shown in figure 1 .

The expression for the strain $\varepsilon_{33}^{\prime}$ along the plane normal is obtained by following the same procedure as given by Singh and Balasingh (1994).

$$
\begin{aligned}
& d_{i j}^{\prime}=a_{i k} a_{j l} d_{k l}, \\
& d_{i j}^{\prime \prime}=b_{i k} b_{j l} d_{k l}^{\prime} .
\end{aligned}
$$

The single and double primes indicate that the quantity is referred to the $x_{i}^{\prime}$ and $x_{i}^{\prime \prime}$ axes respectively. The transformation matrices $a_{i j}$ and $b_{i j}$ are given in an earlier paper (Singh 


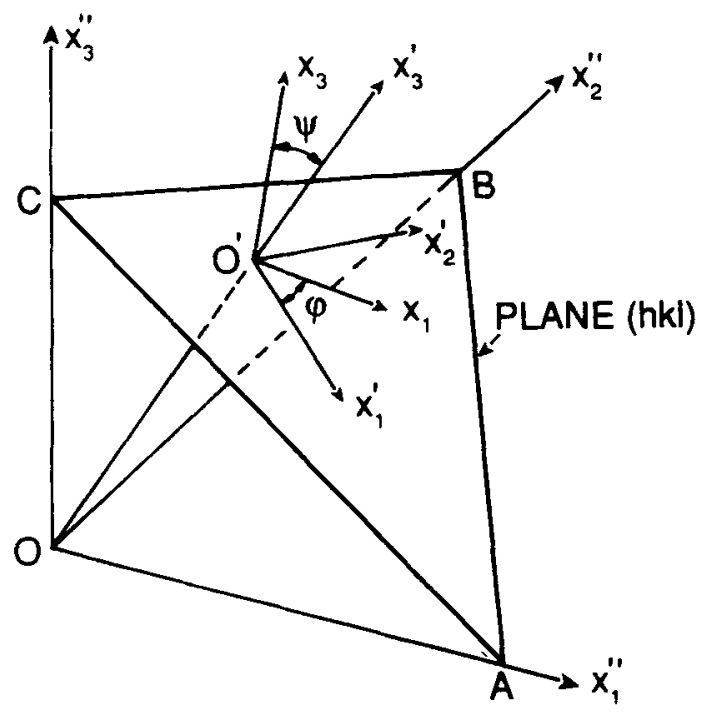

Figure 1. The relative orientations of the three sets of axes.

and Balasingh 1994). Since the elastic constants for trigonal crystals are defined with respect to hexagonal system of axes (Nye 1960), we use the same set of axes to derive the equations in this paper. On carrying out the transformations (5) and (6) and averaging over the $\varphi$-group of crystallites, the following relationships for the deviatoric stress components are obtained.

$$
\begin{aligned}
& d_{11}^{\prime \prime}=\frac{1}{2}\left(1-3 l_{1}^{2}\right) E, \\
& d_{22}^{\prime \prime}=\frac{1}{2}\left(1-3 l_{2}^{2}\right) E, \\
& d_{33}^{\prime \prime}=\frac{1}{2}\left(1-3 l_{3}^{2}\right) E, \\
& d_{23}^{\prime \prime}=-\frac{3}{2} l_{2} l_{3} E, \\
& d_{31}^{\prime \prime}=-\frac{3}{2} l_{3} l_{1} E, \\
& d_{12}^{\prime \prime}=-\frac{3}{2} l_{1} l_{2} E,
\end{aligned}
$$

where

$$
\begin{aligned}
& l_{1}=\frac{\sqrt{3} c h}{M}, \quad l_{2}=\frac{c(h+2 k)}{M}, \quad l_{3}=\frac{\sqrt{3} a l}{M}, \\
& M^{2}=4 c^{2}\left(h^{2}+h k+k^{2}\right)+3 a^{2} l^{2}
\end{aligned}
$$

and

$$
E=\left(\frac{t}{3}\right)\left(1-3 \cos ^{2} \psi\right)
$$

The resulting strain $\varepsilon_{i j}^{\prime \prime}$ is given by

$$
\varepsilon_{i j}^{\prime \prime}=S_{i j k l} d_{k l}^{\prime \prime}
$$


where $S_{i j k l}$ are the elastic compliances at a pressure $\sigma_{\mathrm{p}}$. The strain component along the plane normal is given by

$$
\varepsilon_{33}^{\prime}=\varepsilon_{i j}^{\prime \prime} l_{i} l_{j}
$$

$l_{i}$ are the direction cosines of the $x_{3}^{\prime}$ axis with the $x_{i}^{\prime \prime}$ axes. On carrying out steps (7) and (8), the strain $\varepsilon_{33}^{\prime}$ comes out as follows:

$$
\varepsilon_{33}^{\prime}=\varepsilon_{d}^{R}(h k l)=-\frac{t}{3}\left(1-3 \cos ^{2} \psi\right) /\left(2 G_{\mathrm{R}}^{X}\right)
$$

where

$$
\begin{aligned}
\left(G_{\mathrm{R}}^{X}\right)^{-1}= & \left(2 S_{11}-S_{12}-S_{13}\right)+l_{3}^{2}\left(-5 S_{11}+S_{12}+5 S_{13}-S_{33}+3 S_{44}\right) \\
& +l_{3}^{4}\left(3 S_{11}-6 S_{13}+3 S_{33}-3 S_{44}\right) \\
& +6 l_{2} l_{3}\left(3 l_{1}^{2}-l_{2}^{2}\right) S_{14}+6 l_{1} l_{3}\left(3 l_{2}^{2}-l_{1}^{2}\right) S_{25} .
\end{aligned}
$$

$S_{m n}$ are the elastic compliances in the two-suffix notation at a pressure $\sigma_{p}$. The above equation is for trigonal crystals of classes 3 and $\overline{3}$ which have seven independent elastic compliances. For the crystals of classes $32, \overline{3} \mathrm{~m}$ and $3 \mathrm{~m}$, there are only six independent elastic constants, $S_{25}$ being zero. $\varepsilon_{d}^{R}(h k l)$ represents the lattice strain along the direction $[\mathrm{hkl}]$ produced by the deviatoric stress components under Reuss limit.

\subsection{Derivation of $G_{V}$}

The expression for $\varepsilon_{33}^{\prime}$ under strain continuity or Voigt limit can be derived by writing the expression for $\varepsilon_{33}^{\prime}$ for an elastically isotropic case, and substituting for the elastic constants the Voigt average values. This gives

$$
\varepsilon_{d}^{\mathbf{v}}=-\frac{t}{3}\left(1-3 \cos ^{2} \psi\right) /\left(2 G_{\mathrm{V}}\right)
$$

where $G_{\mathrm{V}}$ is the shear modulus under the Voigt limit, and is given by

$$
G_{\mathrm{V}}=\frac{1}{15}\left[\frac{5}{2}\left(C_{11}-C_{12}\right)+\left(C_{11}-C_{13}\right)+\left(C_{33}-C_{13}\right)+6 C_{44}\right]
$$

where $C_{i j}$ are the elastic stiffnesses in the two-suffix notation at pressure $\sigma_{p}$.

\section{Discussion}

Equation (3) is a generalized expression which holds good for all crystal systems. Only the values of $G_{\mathrm{R}}^{X}$ and $G_{\mathrm{V}}$ differ from one crystal system to another.

Equation (4) shows interesting dependence of $d_{p+d}^{\text {obs }}$ on $\psi$. If $d$-spacings could be measured under non-hydrostatic conditions for at least two $\psi$-values, then $d_{p}$ and $F$ can be determined. Such measurements are possible, for example, in an experimental set-up which is essentially a modification, suggested earlier (Singh 1994), of Kinsland-Bassett geometry (Kinsland and Bassett 1976). The $d$-spacing versus $\psi$-data, however, can be obtained elegantly using a novel technique developed recently by Mao et al (1995).

An examination of $(9)$ shows that for reflections of the type $(00 l)$, the coefficients of 
$S_{14}$ and $S_{25}$ become zero and the expression reduces to the one for hexagonal system. For reflections of the type $(h k 0), \varepsilon_{d}^{\mathrm{R}}(h k l)$ becomes independent of $(h k l)$.

\section{Acknowledgement}

One of the authors (AKS) thanks Dr Ho-kwang Mao whose correspondence in 1995 brought out the necessity of analyzing the non-hydrostatic pressure effect in the trigonal system.

\section{References}

Kinsland G L and Bassett W A 1976 Rev. Sci. Instrum. 47130

Mao Ho-kwang, Shu J. Fei Y, Hu J and Hemley R J 1995 The Wustite enigma (Private communication)

Nye J F 1960 Physical properties of crystals (London. UK: Oxford University Press)

Singh A K 1993 J. Appl. Phys. 73 4278; 745920

Singh A K 1994 High-pressure science and technology-1993; AIP Conf. Proc. (New York: American Institute of Physics) No. 309, p. 1629

Singh A K and Balasingh C 1994 J. Appl. Phys. 754956 